\title{
Contact angle of a hemispherical bubble: an analytical approach
}

Article

Accepted Version

Teixeira, M. A. C. and Teixeira, P. I. C. (2009) Contact angle of a hemispherical bubble: an analytical approach. Journal of Colloid and Interface Science, 338 (1). pp. 193-200. ISSN 0021-9797 doi: https://doi.org/10.1016/j.jcis.2009.05.062 Available at https://centaur.reading.ac.uk/29242/

It is advisable to refer to the publisher's version if you intend to cite from the work. See Guidance on citing.

Published version at: http://dx.doi.org/10.1016/j.jcis.2009.05.062

To link to this article DOI: http://dx.doi.org/10.1016/j.jcis.2009.05.062

Publisher: Elsevier

All outputs in CentAUR are protected by Intellectual Property Rights law, including copyright law. Copyright and IPR is retained by the creators or other copyright holders. Terms and conditions for use of this material are defined in the End User Agreement.

\section{www.reading.ac.uk/centaur}

\section{CentAUR}

Central Archive at the University of Reading

Reading's research outputs online 


\title{
Contact angle of a hemispherical bubble: an analytical approach
}

\author{
M. A. C. Teixeira ${ }^{1}$ and P. I. C. Teixeira ${ }^{2,3}$ \\ ${ }^{1}$ University of Lisbon, CGUL, IDL, \\ Edifício C8, Campo Grande, P-1749-016 Lisbon, Portugal \\ ${ }^{2}$ Instituto Superior de Engenharia de Lisboa, \\ Rua Conselheiro Emídio Navarro, P-1950-062 Lisbon, Portugal \\ ${ }^{3}$ Centro de Física Teórica e Computacional, University of Lisbon, \\ Avenida Professor Gama Pinto 2, P-1649-003 Lisbon, Portugal
}

(Dated: 25 May 2009)

\begin{abstract}
We have calculated the equilibrium shape of the axially symmetric Plateau border along which a spherical bubble contacts a flat wall, by analytically integrating Laplace's equation in the presence of gravity, in the limit of small Plateau border sizes. This method has the advantage that it provides closed-form expressions for the positions and orientations of the Plateau border surfaces. Results are in very good overall agreement with those obtained from a numerical solution procedure, and are consistent with experimental data. In particular we find that the effect of gravity on Plateau border shape is relatively small for typical bubble sizes, leading to a widening of the Plateau border for sessile bubbles and to a narrowing for pendant bubbles. The contact angle of the bubble is found to depend even more weakly on gravity.
\end{abstract}

PACS numbers: 47.57.Bc, 47.55.np 


\section{INTRODUCTION}

A liquid foam is an assembly of gas bubbles bounded by liquid films. Foams are encountered in many practical applications such as beverages, toiletries, cleaning products, fire fighting, oil recovery, mixture fractionation, the manufacture of cellular materials, and ore purification by flotation [1]. The behaviour of a foam with a low-viscosity liquid phase (e.g., an aqueous foam or a metal foam, as opposed to a polymeric foam) is dominated by surface tension. Such foams thus serve as models for systems in which the interfacial area (in three dimensions $(3 \mathrm{D})$ ) or the perimeter (in two dimensions (2D)) is minimised at equilibrium. In the limit of a perfectly dry foam, such as may be obtained after drainage of most of its liquid content due to gravity, the films can be approximated as surfaces of zero thickness endowed with a contractile tendency that is described by a film tension, denoted $2 \gamma$ (a free energy per unit length of a 2D film, or per unit area of a 3D film, which is twice that of the liquid-vapour interface, $\gamma$ ). At equilibrium a dry foam satisfies Plateau's laws [2]: films of constant mean curvature meet in triple lines at $2 \pi / 3$ angles; the triple lines meet in fourfold vertices at the tetrahedral angles; and the different pressures in the bubbles equilibrate the contractile forces on the films. The energy of such a foam is just the energy of its films.

In actual moderately dry foams (liquid content below about 5\%), we may still neglect the film thicknesses (of order $100 \mathrm{~nm}$ ), but the triple lines are 'decorated' with regions of triangular cross-section called Plateau borders (of width of order $0.1-1 \mathrm{~mm}$ ) where most of the liquid resides. In addition, where a foam meets a confining surface there are wall Plateau borders. These are bounded by two liquid surfaces of tension $\gamma$ and one solid surface (the wall) of tension $\gamma_{W L}$ (the wall-liquid interfacial tension). Wall Plateau borders affect both the statics and the dynamics of foams: not only do they contribute to the total foam energy, they also exert considerable drag on the walls in foam flow experiments. In perfectly dry foams the film contact angle at a wall is $\pi / 2$. In $2 \mathrm{D}$ wet foams, the (circular) film prolongations into a wall PB also meet the wall at $\pi / 2$ [3]. However, this appears not to be the case in $3 \mathrm{D}$ wet foams in contact with walls: deviations from $\pi / 2$ have been reported for a single bubble on a wet porous substrate [4], e.g., $\phi \approx 85^{\circ}$ (measured inside the bubble and extrapolated to the substrate surface - see figure 1) for a bubble of radius $R=2.4 \mathrm{~mm}$. The reason is that the Plateau border possesses curvature in the horizontal direction, due to the axial symmetry of the bubble. Although generally weaker than that existing in the 
vertical direction, this curvature depends on bubble and Plateau border size, and modifies the contact angle of the bubble.

In an earlier paper [5] the Plateau border shapes and the apparent contact angle of a single bubble at a wall were calculated by numerical integration of the appropriate Laplace equation. The usual disparity of scales between the two curvatures, however, suggests the use of perturbation methods, which would allow greater insight into the physical mechanism involved. Taking into account that, in most practical situations, the height of the Plateau border $h$ is considerably smaller than the radius of the bubble $R$, in this paper we develop an approximate analytical solution for the Plateau border shape in powers of $h / R$. This solution will also be used to study the relatively weak gravity effects on the Plateau border (i.e., the difference between sessile and pendant bubbles). Gravity is important in many aspects of foam research, e.g., drainage [1], and assumes ever greater relevance as it becomes increasingly possible to carry out experiments in microgravity environments, e.g., aboard the International Space Station. The analytical approach used in the present study has the advantage of allowing a better control of input conditions, and consequently an easier and quicker exploration of parameter space, than the numerical model used in [5] (where gravity effects were not addressed).

This paper is organised as follows: in section II we describe our model, the Laplace equation for the Plateau border around a single spherical bubble at a flat wall, and obtain closed-form expressions for the inner and outer Plateau border surfaces. Our results for the apparent contact angle, extrapolated contact angle and Plateau border shape are discussed in section III: we compare results at different orders in $h / R$ with those from numerical solution of the Laplace equation, for different contact angles of the liquid on the substrate and varying gravity strengths. Comparison is also made with what is, to our knowledge, the only existing set of experimental results for this system [4]. Finally, section IV contains some concluding remarks. 


\section{THEORETICAL MODEL}

The starting point is Laplace's equation for an axisymmetric geometry, which may be written:

$$
\left[1+\left(\frac{d x}{d z}\right)^{2}\right]^{-3 / 2}\left[-\frac{d^{2} x}{d z^{2}}+\frac{1+\left(\frac{d x}{d z}\right)^{2}}{x}\right]=\frac{\Delta p}{\gamma},
$$

where $z$ is the height and $x$ is the distance between the film surface and its axis of symmetry (here assumed to be the $z$ axis). $\Delta p$ is the pressure difference across the film surface (inner minus outer) and $\gamma$ is the surface tension of the fluid under consideration.

Defining $\cot \theta=-d x / d z, \theta$ is the angle, measured on a vertical plane containing the $z$ axis, between the film direction and the horizontal direction. The contact angle at which the outer surface of the Plateau border intersects the substrate at the bottom of the bubble will be called $\theta_{1}=\theta(z=0)$, while the corresponding contact angle of the inner surface is, of course, $\pi-\theta_{1}$. Finally, the apparent contact angle of the bubble, defined as the angle of the film at the top of the Plateau border, where both surfaces are tangent, is called $\theta_{2}=\theta(z=h)$ (see figure 1). Replacing the dependent variable $x$ by $\theta$ in equation (1), that equation takes the form

$$
\left(-\frac{d \theta}{d z}+\frac{1}{R \sin \theta_{2}+\int_{z}^{h} \cot \theta d z}\right) \sin \theta=\frac{\Delta p}{\gamma} .
$$

Applying equation (2) at the inner and outer surfaces of the Plateau border yields

$$
\begin{gathered}
\left(-\frac{d \theta}{d z}+\frac{1}{R \sin \theta_{2}+\int_{z}^{h} \cot \theta d z}\right) \sin \theta=\frac{p_{i}-p_{b}}{\gamma} \quad \text { (inner) } \\
\left(\frac{d \theta}{d z}-\frac{1}{R \sin \theta_{2}+\int_{z}^{h} \cot \theta d z}\right) \sin \theta=\frac{p_{o}-p_{b}}{\gamma} \quad \text { (outer) }
\end{gathered}
$$

where $p_{i}, p_{o}$ and $p_{b}$ are the pressures inside the bubble, outside the bubble, and inside the Plateau border, respectively. The pressure inside the Plateau border is assumed to be in hydrostatic equilibrium, such that

$$
p_{b}=p_{b 0}-\rho g z
$$

where $p_{b 0}$ is the pressure at the bottom of the Plateau border, $g$ is the acceleration of gravity and $\rho$ is the density of the fluid under consideration. Additionally, it should be noted that the pressure difference between the inside and the outside of the bubble is given by

$$
p_{i}-p_{o}=4 \frac{\gamma}{R}
$$


In their present form, equations (3)-(4) cannot be solved analytically. In order to make this possible, they are first inverted, so that $z$ becomes the dependent variable and $\theta$ the independent variable. To do this, it is necessary to change the variable of integration of the integral from $z$ to $\theta$, on noting that, at the surfaces bounding the Plateau border, $\theta$ is a monotonic function of $z$ :

$$
\int_{z}^{h} \cot \theta d z=\int_{\theta}^{\theta_{2}} \cot \theta \frac{d z}{d \theta} d \theta
$$

When this is done, and equation (5) is also taken into account, equations (3)-(4) take the form

$$
\begin{aligned}
& \left(\frac{p_{i}-p_{b 0}}{\gamma}+\frac{\rho g z}{\gamma}-\frac{\sin \theta}{R \sin \theta_{2}+\int_{\theta}^{\theta_{2}} \cot \theta \frac{d z}{d \theta} d \theta}\right) \frac{d z}{d \theta}=-\sin \theta \quad \text { (inner) } \\
& \left(\frac{p_{o}-p_{b 0}}{\gamma}+\frac{\rho g z}{\gamma}+\frac{\sin \theta}{R \sin \theta_{2}+\int_{\theta}^{\theta_{2}} \cot \theta \frac{d z}{d \theta} d \theta}\right) \frac{d z}{d \theta}=\sin \theta \quad \text { (outer). }
\end{aligned}
$$

At this point, it is useful to make the variables of this problem dimensionless, so that the orders of magnitude of the various terms become clearer. $\theta$ is dimensionless by nature, and takes values of $O(1)$. Since these equations are going to be integrated over the Plateau border height, which is $h$, a normalised height is defined as $z^{\prime}=z / h$. Then equations (8)-(9) become

$$
\begin{aligned}
& {\left[\frac{\left(p_{i}-p_{b 0}\right) h}{\gamma}+\frac{h^{2}}{R^{2}} \frac{\rho g R^{2}}{\gamma} z^{\prime}-\frac{h}{R} \frac{\sin \theta}{\sin \theta_{2}\left(1+\frac{h}{R} \frac{1}{\sin \theta_{2}} \int_{\theta}^{\theta_{2}} \cot \theta \frac{d z^{\prime}}{d \theta} d \theta\right)}\right] \frac{d z^{\prime}}{d \theta}=-\sin \theta \quad \text { (inner), }} \\
& {\left[\frac{\left(p_{o}-p_{b 0}\right) h}{\gamma}+\frac{h^{2}}{R^{2}} \frac{\rho g R^{2}}{\gamma} z^{\prime}+\frac{h}{R} \frac{\sin \theta}{\sin \theta_{2}\left(1+\frac{h}{R} \frac{1}{\sin \theta_{2}} \int_{\theta}^{\theta_{2}} \cot \theta \frac{d z^{\prime}}{d \theta} d \theta\right)}\right] \frac{d z^{\prime}}{d \theta}=\sin \theta \quad \text { (outer).(11) }}
\end{aligned}
$$

These equations are subject to the lower boundary condition $z^{\prime}\left(\theta=\pi-\theta_{1}\right)=0$ (at the inner surface) or $z^{\prime}\left(\theta=\theta_{1}\right)=0$ (at the outer surface) and the upper boundary condition $z^{\prime}\left(\theta=\theta_{2}\right)=1$ (at both surfaces).

Noting that $h / R$ is small, when the factor involving the integral is expanded in powers of $h / R$ up to first order, equations (10)-(11) can be written approximately as

$$
\begin{aligned}
& {\left[\frac{\left(p_{i}-p_{b 0}\right) h}{\gamma}+\frac{h^{2}}{R^{2}} \frac{\rho g R^{2}}{\gamma} z^{\prime}-\frac{h}{R} \frac{\sin \theta}{\sin \theta_{2}}\left(1-\frac{h}{R} \frac{1}{\sin \theta_{2}} \int_{\theta}^{\theta_{2}} \cot \theta \frac{d z^{\prime}}{d \theta} d \theta\right)\right] \frac{d z^{\prime}}{d \theta}=-\sin \theta \quad \text { (inner), }} \\
& {\left[\frac{\left(p_{o}-p_{b 0}\right) h}{\gamma}+\frac{h^{2}}{R^{2}} \frac{\rho g R^{2}}{\gamma} z^{\prime}+\frac{h}{R} \frac{\sin \theta}{\sin \theta_{2}}\left(1-\frac{h}{R} \frac{1}{\sin \theta_{2}} \int_{\theta}^{\theta_{2}} \cot \theta \frac{d z^{\prime}}{d \theta} d \theta\right)\right] \frac{d z^{\prime}}{d \theta}=\sin \theta \quad \text { (outer). }}
\end{aligned}
$$


In equations (12)-(13), it is clear that only the first term in square brackets is of $O(1)$, to balance the right-hand side of these equations, while the third term, related to the horizontal curvature of the Plateau border, is of $O(h / R)$. The last term, related to the fact that the inner surface of the Plateau border is closer to the axis of symmetry than the outer surface, is of $O(h / R)^{2}$. Finally, the second term, related to gravity effects, is also of $O(h / R)^{2}$. In fact, whether this term is of second order or not depends on the value of the dimensionless parameter $\rho g R^{2} / \gamma$, but in the cases that will be addressed - bubbles of radius $R \approx 1-3 \mathrm{~mm}$, as in [4] - this parameter is indeed of $O(1)$, as required. For these values of $R$ and using $\gamma=33.6 \times 10^{-3} \mathrm{~J} \mathrm{~m}^{-2}$ (see [4]) gives $\rho g R^{2} / \gamma \approx 0.3-2.6$.

The problem is tackled by expanding both the dimensionless height $z^{\prime}(\theta)$ and the dimensionless inner and outer pressures in power series of $h / R$, as follows:

$$
\begin{aligned}
z^{\prime} & =z_{0}^{\prime}+\left(\frac{h}{R}\right) z_{1}^{\prime}+\left(\frac{h}{R}\right)^{2} z_{2}^{\prime}+\ldots, \\
\frac{\left(p_{i}-p_{b 0}\right) h}{\gamma} & =p_{i 0}^{\prime}+\left(\frac{h}{R}\right) p_{i 1}^{\prime}+\left(\frac{h}{R}\right)^{2} p_{i 2}^{\prime}+\ldots, \\
\frac{\left(p_{o}-p_{b 0}\right) h}{\gamma} & =p_{o 0}^{\prime}+\left(\frac{h}{R}\right) p_{o 1}^{\prime}+\left(\frac{h}{R}\right)^{2} p_{o 2}^{\prime}+\ldots
\end{aligned}
$$

Although a similar power series solution for $\theta$ could presumably be used to solve the equations before inversion (i.e., equations (3) and (4)), calculations would certainly be less straightforward, because $\theta$ appears as the argument of sine and cosine functions. Additionally, this would necessarily impose an a-priori dependence of $\theta_{2}$ on $h / R$ (quadratic for a second-order expansion) which, as will be seen, is not supported by the numerical simulations. The solution procedure will first be described for the inner surface of the Plateau border.

\section{A. Inner surface}

Once equations (14)-(15) are inserted into equation (12), three equations result, valid at zeroth-, first- and second-order in $h / R$, respectively:

$$
\begin{aligned}
& p_{i 0}^{\prime} \frac{d z_{0}^{\prime}}{d \theta}=-\sin \theta \\
& p_{i 0}^{\prime} \frac{d z_{1}^{\prime}}{d \theta}+\left(p_{i 1}^{\prime}-\frac{\sin \theta}{\sin \theta_{2}}\right) \frac{d z_{0}^{\prime}}{d \theta}=0 \\
& p_{i 0}^{\prime} \frac{d z_{2}^{\prime}}{d \theta}+\left(p_{i 1}^{\prime}-\frac{\sin \theta}{\sin \theta_{2}}\right) \frac{d z_{1}^{\prime}}{d \theta}+\left(p_{i 2}^{\prime}+\frac{\rho g R^{2}}{\gamma} z_{0}^{\prime}+\frac{\sin \theta}{\sin ^{2} \theta_{2}} \int_{\theta}^{\theta_{2}} \cot \theta \frac{d z_{0}^{\prime}}{d \theta} d \theta\right) \frac{d z_{0}^{\prime}}{d \theta}=0
\end{aligned}
$$


These equations must be solved subject to the boundary conditions $z_{0}^{\prime}\left(\theta=\pi-\theta_{1}\right)=z_{1}^{\prime}(\theta=$ $\left.\pi-\theta_{1}\right)=z_{2}^{\prime}\left(\theta=\pi-\theta_{1}\right)=0$ and $z_{0}^{\prime}\left(\theta=\theta_{2}\right)=1, z_{1}^{\prime}\left(\theta=\theta_{2}\right)=z_{2}^{\prime}\left(\theta=\theta_{2}\right)=0$. Integrating equations (17)-(19) between $\theta=\pi-\theta_{1}$ and $\theta=\theta_{2}$ using these boundary conditions yields the following expansion coefficients of the inner pressure:

$$
\begin{aligned}
& p_{i 0}^{\prime}=\cos \theta_{1}+\cos \theta_{2}, \\
& p_{i 1}^{\prime}=-\frac{1}{2} \frac{\theta_{2}-\pi+\theta_{1}-\sin \theta_{2} \cos \theta_{2}-\sin \theta_{1} \cos \theta_{1}}{p_{i 0}^{\prime} \sin \theta_{2}}, \\
& p_{i 2}^{\prime}=-\frac{1}{2} \frac{\rho g R^{2}}{\gamma}+\frac{p_{i 1}^{\prime}}{p_{i 0}^{\prime}}-\frac{p_{i 1}^{\prime 2}}{p_{i 0}^{\prime}} .
\end{aligned}
$$

In fact, as will be seen later, one does not need to find explicit expressions for $z_{0}^{\prime}, z_{1}^{\prime}$ and $z_{2}^{\prime}$ in order to obtain a relation between $\theta_{1}, \theta_{2}$ and $h / R$. However, this is necessary for plotting the actual shapes of the Plateau border surfaces. To obtain such expressions, equations (17)-(19) must be integrated instead between $\theta=\pi-\theta_{1}$ and a generic $\theta$, with the result

$$
\begin{aligned}
z_{0}^{\prime}= & \frac{\cos \theta+\cos \theta_{1}}{p_{i 0}^{\prime}} \\
z_{1}^{\prime}= & -\frac{p_{i 1}^{\prime}}{p_{i 0}^{\prime 2}}\left(\cos \theta+\cos \theta_{1}\right)-\frac{1}{2 p_{i 0}^{\prime 2} \sin \theta_{2}}\left(\theta-\pi+\theta_{1}-\sin \theta \cos \theta-\sin \theta_{1} \cos \theta_{1}\right) \\
z_{2}^{\prime}= & -\frac{1}{2} \frac{\rho g R^{2}}{\gamma} \frac{\left(\cos \theta+\cos \theta_{1}\right)^{2}}{p_{i 0}^{\prime 3}}-\left(\frac{p_{i 2}^{\prime}}{p_{i 0}^{\prime 2}}-\frac{p_{i 1}^{\prime 2}}{p_{i 0}^{\prime 3}}\right)\left(\cos \theta+\cos \theta_{1}\right) \\
& +\left(\frac{p_{i 1}^{\prime}}{p_{i 0}^{\prime 3}}-\frac{1}{2 p_{i 0}^{\prime 3}}\right) \frac{\theta-\pi+\theta_{1}-\sin \theta \cos \theta-\sin \theta_{1} \cos \theta_{1}}{\sin \theta_{2}} .
\end{aligned}
$$

In order to specify the inner surface of the Plateau border, it is also necessary to know its horizontal position. An equation analogous to equation (12) can be obtained if we define $x^{\prime}=x / h$ and $\Delta x^{\prime}=x^{\prime}\left(\theta=\theta_{2}\right)-x^{\prime}(\theta)$ (where $x$ is the horizontal coordinate of the inner surface). From these definitions it follows that, although $x^{\prime}$ is of $O(R / h), \Delta x^{\prime}$ is of $O(1)$. If we note that $d x^{\prime} / d z^{\prime}=-\cot \theta$, then it can be shown from equation (12) that

$$
\left[\frac{\left(p_{i}-p_{b 0}\right) h}{\gamma}+\frac{h^{2}}{R^{2}} \frac{\rho g R^{2}}{\gamma} z^{\prime}-\frac{h}{R} \frac{\sin \theta}{\sin \theta_{2}}\left(1+\frac{h}{R} \frac{1}{\sin \theta_{2}} \Delta x^{\prime}\right)\right] \frac{d \Delta x^{\prime}}{d \theta}=-\cos \theta .
$$

If $\Delta x^{\prime}$ is expanded in a power series of $h / R$, as follows,

$$
\Delta x^{\prime}=\Delta x_{0}^{\prime}+\left(\frac{h}{R}\right) \Delta x_{1}^{\prime}+\left(\frac{h}{R}\right)^{2} \Delta x_{2}^{\prime}+\ldots,
$$

and equations (14)-(15) are also taken into account, three equations for $\Delta x_{0}^{\prime}, \Delta x_{1}^{\prime}$ and $\Delta x_{2}^{\prime}$ follow:

$$
p_{i 0}^{\prime} \frac{d \Delta x_{0}^{\prime}}{d \theta}=-\cos \theta
$$




$$
\begin{aligned}
& p_{i 0}^{\prime} \frac{d \Delta x_{1}^{\prime}}{d \theta}+\left(p_{i 1}^{\prime}-\frac{\sin \theta}{\sin \theta_{2}}\right) \frac{d \Delta x_{0}^{\prime}}{d \theta}=0 \\
& p_{i 0}^{\prime} \frac{d \Delta x_{2}^{\prime}}{d \theta}+\left(p_{i 1}^{\prime}-\frac{\sin \theta}{\sin \theta_{2}}\right) \frac{d \Delta x_{1}^{\prime}}{d \theta}+\left(p_{i 2}^{\prime}+\frac{\rho g R^{2}}{\gamma} z_{0}^{\prime}-\frac{\sin \theta}{\sin ^{2} \theta_{2}} \Delta x_{0}^{\prime}\right) \frac{d \Delta x_{0}^{\prime}}{d \theta}=0 .
\end{aligned}
$$

The solutions to these equations satisfying the boundary conditions $\Delta x_{0}^{\prime}\left(\theta=\theta_{2}\right)=\Delta x_{1}^{\prime}(\theta=$ $\left.\theta_{2}\right)=\Delta x_{2}^{\prime}\left(\theta=\theta_{2}\right)=0$ (which result from the definition of $\Delta x^{\prime}$ and equation (27)), are:

$$
\begin{aligned}
\Delta x_{0}^{\prime}= & \frac{\sin \theta_{2}-\sin \theta}{p_{i 0}^{\prime}} \\
\Delta x_{1}^{\prime}= & \frac{1}{2 p_{i 0}^{\prime 2} \sin \theta_{2}}\left(\sin ^{2} \theta_{2}-\sin ^{2} \theta\right)-\frac{p_{i 1}^{\prime}}{p_{i 0}^{\prime 2}}\left(\sin \theta_{2}-\sin \theta\right) \\
\Delta x_{2}^{\prime}= & \left(\frac{1}{2 p_{i 0}^{\prime 3}}-\frac{p_{i 1}^{\prime}}{p_{i 0}^{\prime 3}}\right) \frac{\sin ^{2} \theta_{2}-\sin ^{2} \theta}{\sin \theta_{2}}+\left(\frac{p_{i 1}^{\prime 2}}{p_{i 0}^{\prime 3}}-\frac{p_{i 2}^{\prime}}{p_{i 0}^{\prime 2}}-\frac{\rho g R^{2}}{\gamma} \frac{\cos \theta_{1}}{p_{i 0}^{\prime 3}}\right)\left(\sin \theta_{2}-\sin \theta\right) \\
& -\frac{\rho g R^{2}}{\gamma} \frac{1}{2 p_{i 0}^{\prime 3}}\left(\theta_{2}-\theta+\sin \theta_{2} \cos \theta_{2}-\sin \theta \cos \theta\right)
\end{aligned}
$$

This completely specifies the inner surface of the Plateau border.

\section{B. Outer surface}

Now the same procedure must be followed for the outer surface of the Plateau border. Hence, from equations (14), (16) and (13), three equations result, again valid at zeroth-, first- and second-order in $h / R$, respectively:

$$
\begin{aligned}
& p_{o 0}^{\prime} \frac{d z_{0}^{\prime}}{d \theta}=\sin \theta \\
& p_{o 0}^{\prime} \frac{d z_{1}^{\prime}}{d \theta}+\left(p_{o 1}^{\prime}+\frac{\sin \theta}{\sin \theta_{2}}\right) \frac{d z_{0}^{\prime}}{d \theta}=0 \\
& p_{o 0}^{\prime} \frac{d z_{2}^{\prime}}{d \theta}+\left(p_{o 1}^{\prime}+\frac{\sin \theta}{\sin \theta_{2}}\right) \frac{d z_{1}^{\prime}}{d \theta}+\left(p_{o 2}^{\prime}+\frac{\rho g R^{2}}{\gamma} z_{0}^{\prime}-\frac{\sin \theta}{\sin ^{2} \theta_{2}} \int_{\theta}^{\theta_{2}} \cot \theta \frac{d z_{0}^{\prime}}{d \theta} d \theta\right) \frac{d z_{0}^{\prime}}{d \theta}=0
\end{aligned}
$$

These equations are subject to the boundary conditions $z_{0}^{\prime}\left(\theta=\theta_{1}\right)=z_{1}^{\prime}\left(\theta=\theta_{1}\right)=z_{2}^{\prime}(\theta=$ $\left.\theta_{1}\right)=0$ and $z_{0}^{\prime}\left(\theta=\theta_{2}\right)=1, z_{1}^{\prime}\left(\theta=\theta_{2}\right)=z_{2}^{\prime}\left(\theta=\theta_{2}\right)=0$. Integrating them between $\theta=\theta_{1}$ and $\theta=\theta_{2}$ gives

$$
\begin{aligned}
& p_{o 0}^{\prime}=\cos \theta_{1}-\cos \theta_{2} \\
& p_{o 1}^{\prime}=-\frac{1}{2} \frac{\theta_{2}-\theta_{1}-\sin \theta_{2} \cos \theta_{2}+\sin \theta_{1} \cos \theta_{1}}{p_{o 0}^{\prime} \sin \theta_{2}} \\
& p_{o 2}^{\prime}=-\frac{1}{2} \frac{\rho g R^{2}}{\gamma}-\frac{p_{o 1}^{\prime}}{p_{o 0}^{\prime}}-\frac{p_{o 1}^{\prime 2}}{p_{o 0}^{\prime}}
\end{aligned}
$$


On the other hand, integrating equations (34)-(36) between $\theta=\theta_{1}$ and a generic $\theta$ yields

$$
\begin{aligned}
z_{0}^{\prime}= & \frac{\cos \theta_{1}-\cos \theta}{p_{o 0}^{\prime}}, \\
z_{1}^{\prime}= & -\frac{p_{o 1}^{\prime}}{p_{o 0}^{\prime 2}}\left(\cos \theta_{1}-\cos \theta\right)-\frac{1}{2 p_{o 0}^{\prime 2} \sin \theta_{2}}\left(\theta-\theta_{1}-\sin \theta \cos \theta+\sin \theta_{1} \cos \theta_{1}\right), \\
z_{2}^{\prime}= & -\frac{1}{2} \frac{\rho g R^{2}}{\gamma} \frac{\left(\cos \theta_{1}-\cos \theta\right)^{2}}{p_{o 0}^{\prime 3}}-\left(\frac{p_{o 2}^{\prime}}{p_{o 0}^{\prime 2}}-\frac{p_{o 1}^{\prime 2}}{p_{o 0}^{\prime 3}}\right)\left(\cos \theta_{1}-\cos \theta\right) \\
& +\left(\frac{p_{o 1}^{\prime}}{p_{o 0}^{\prime 3}}+\frac{1}{2 p_{o 0}^{\prime 3}}\right) \frac{\theta-\theta_{1}-\sin \theta \cos \theta+\sin \theta_{1} \cos \theta_{1}}{\sin \theta_{2}} .
\end{aligned}
$$

An equation analogous to equation (26) may be obtained for the horizontal displacement of the outer surface, $\Delta x^{\prime}=x^{\prime}\left(\theta=\theta_{2}\right)-x^{\prime}(\theta)$ :

$$
\left[\frac{\left(p_{o}-p_{b 0}\right) h}{\gamma}+\frac{h^{2}}{R^{2}} \frac{\rho g R^{2}}{\gamma} z^{\prime}+\frac{h}{R} \frac{\sin \theta}{\sin \theta_{2}}\left(1+\frac{h}{R} \frac{1}{\sin \theta_{2}} \Delta x^{\prime}\right)\right] \frac{d \Delta x^{\prime}}{d \theta}=\cos \theta .
$$

Expanding $\Delta x^{\prime}$ in powers of $h / R$, as in equation (27), the following three equations are obtained from equation (43):

$$
\begin{aligned}
& p_{o 0}^{\prime} \frac{d \Delta x_{0}^{\prime}}{d \theta}=\cos \theta \\
& p_{o 0}^{\prime} \frac{d \Delta x_{1}^{\prime}}{d \theta}+\left(p_{o 1}^{\prime}+\frac{\sin \theta}{\sin \theta_{2}}\right) \frac{d \Delta x_{0}^{\prime}}{d \theta}=0 \\
& p_{o 0}^{\prime} \frac{d \Delta x_{2}^{\prime}}{d \theta}+\left(p_{o 1}^{\prime}+\frac{\sin \theta}{\sin \theta_{2}}\right) \frac{d \Delta x_{1}^{\prime}}{d \theta}+\left(p_{o 2}^{\prime}+\frac{\rho g R^{2}}{\gamma} z_{0}^{\prime}+\frac{\sin \theta}{\sin ^{2} \theta_{2}} \Delta x_{0}^{\prime}\right) \frac{d \Delta x_{0}^{\prime}}{d \theta}=0 .
\end{aligned}
$$

Subject to the same boundary conditions as enunciated before for the inner surface, these equations have the solutions:

$$
\begin{aligned}
\Delta x_{0}^{\prime}= & -\frac{\sin \theta_{2}-\sin \theta}{p_{o 0}^{\prime}} \\
\Delta x_{1}^{\prime}= & \frac{1}{2 p_{o 0}^{\prime 2} \sin \theta_{2}}\left(\sin ^{2} \theta_{2}-\sin ^{2} \theta\right)+\frac{p_{o 1}^{\prime}}{p_{o 0}^{\prime 2}}\left(\sin \theta_{2}-\sin \theta\right) \\
\Delta x_{2}^{\prime}= & -\left(\frac{1}{2 p_{o 0}^{\prime 3}}+\frac{p_{o 1}^{\prime}}{p_{o 0}^{\prime 3}}\right) \frac{\sin ^{2} \theta_{2}-\sin ^{2} \theta}{\sin \theta_{2}}-\left(\frac{p_{o 1}^{\prime 2}}{p_{o 0}^{\prime 3}}-\frac{p_{o 2}^{\prime}}{p_{o 0}^{\prime 2}}-\frac{\rho g R^{2}}{\gamma} \frac{\cos \theta_{1}}{p_{o 0}^{\prime 3}}\right)\left(\sin \theta_{2}-\sin \theta\right) \\
& -\frac{\rho g R^{2}}{\gamma} \frac{1}{2 p_{o 0}^{\prime 3}}\left(\theta_{2}-\theta+\sin \theta_{2} \cos \theta_{2}-\sin \theta \cos \theta\right) .
\end{aligned}
$$

This specifies the outer surface completely. It should be noted that, by design of the solutions, the upper vertex of the Plateau border, where the inner and outer surfaces meet, is located at $\Delta x^{\prime}=0$ and $z^{\prime}=1$. 


\section{Relation between $\theta_{1}, \theta_{2}$ and $h / R$}

The relation between the inner and outer pressures (due to the bubble curvature) allows us to relate $\theta_{1}, \theta_{2}$ and $h / R$. The dimensionless version of equation (6) is

$$
\frac{\left(p_{i}-p_{o}\right) h}{\gamma}=4 \frac{h}{R}
$$

This equation shows that, although the pressure differences between the inside of the Plateau border and the inside or the outside of the bubble are of zeroth order in $h / R$, the difference between these pressure differences is only of first order. This, of course, is consistent with

the assumed disparity of scales between the curvatures of the Plateau border surfaces and of the bubble. Equation (50), combined with equations (15)-(16), gives the following equation for $h / R$ (accurate to second order in $h / R$ ),

$$
\left(p_{i 2}^{\prime}-p_{o 2}^{\prime}\right)\left(\frac{h}{R}\right)^{2}+\left(p_{i 1}^{\prime}-p_{o 1}^{\prime}-4\right)\left(\frac{h}{R}\right)+p_{i 0}^{\prime}-p_{o 0}^{\prime}=0
$$

which enables us to find $h / R$ as a function of $\theta_{1}$ and $\theta_{2}$, through the solution

$$
\frac{h}{R}=\frac{4-p_{i 1}^{\prime}+p_{o 1}^{\prime}-\sqrt{\left(4-p_{i 1}^{\prime}+p_{o 1}^{\prime}\right)^{2}-4\left(p_{i 2}^{\prime}-p_{o 2}^{\prime}\right)\left(p_{i 0}^{\prime}-p_{o 0}^{\prime}\right)}}{2\left(p_{i 2}^{\prime}-p_{o 2}^{\prime}\right)}
$$

(where the pressures are specified by equations (20)-(22) and (37)-(39)). This formula provides a relation between $h / R, \theta_{2}$ and $\theta_{1}$ accurate to second order in $h / R$. A relation accurate to first-order may be obtained by retaining only the zeroth- and first-order terms in equation (51), which gives:

$$
\frac{h}{R}=\frac{p_{i 0}^{\prime}-p_{o 0}^{\prime}}{4-p_{i 1}^{\prime}+p_{o 1}^{\prime}} .
$$

Finally, a zeroth-order approximation can be obtained by neglecting both the first- and the second-order terms in equation (51), yielding:

$$
p_{i 0}^{\prime}=p_{o 0}^{\prime} \quad \Rightarrow \quad \cos \theta_{2}=0 \quad \Rightarrow \quad \theta_{2}=90^{\circ} .
$$

Note that in equation (52) the physically meaningful root, i.e., the one that reduces to the first-order approximation as $p_{i 2}^{\prime}$ and $p_{o 2}^{\prime}$ tend to zero, has been selected.This can be checked by rewriting equation $(52)$ as

$$
\frac{h}{R}=\frac{\left(4-p_{i 1}^{\prime}+p_{o 1}^{\prime}\right)\left[1-\sqrt{1-\frac{4\left(p_{i 1}^{\prime}-p_{o 1}^{\prime}\right)\left(p_{i 2}^{\prime}-p_{o 2}^{\prime}\right)}{\left(4-p_{i 1}^{\prime}+p_{o 1}^{\prime}\right)^{2}}}\right]}{2\left(p_{i 2}^{\prime}-p_{o 2}^{\prime}\right)}
$$


and expanding the term inside the square brackets in a Taylor series.

Note also that the procedure described above is not part of the formal power series solution to the problem (where the coefficients multiplying successive powers of $h / R$ would have to be set to zero to satisfy equation (51)). In fact, these coefficients have been determined previously in equations (20)-(22) and (37)-(39), so equation (51) is not valid for any arbitrary $h / R$, but rather defines $h / R$ as a function of $\theta_{1}$ and $\theta_{2}$. Equation (51) and its versions truncated at lower orders, which give rise to the solutions of equations (52), (53) or (54), may be viewed as akin to truncating the final result of a perturbation expansion solution at the required order.

In equation (51), and unlike what is usual in perturbation expansions, what is determined is the small parameter $h / R$ as a function of other problem variables, instead of the other way round. This was done for simplicity but we believe the procedure to be consistent, as the ensuing results will show, despite the fact that it is not very standard. Obviously, solutions analogous to equation (52) would be either much lengthier or impossible to obtain analytically using this approach if the perturbation expansion was extended to third or higher order.

\section{RESULTS}

\section{A. Apparent contact angle of the bubble}

Figure 2(a,b) shows the variation of the apparent contact angle of the bubble $\theta_{2}$ with $h / R$ for three values of $\theta_{1}$ (the contact angle between the Plateau border surfaces and the substrate). Results in figure 2(a) are for zero gravity and those in figure 2(b) for two values of the gravity parameter (positive and negative, corresponding to sessile and pendant bubbles, respectively). In figure $2(\mathrm{a})$, the zeroth-order solution, equation (54), is coincident with the upper horizontal axis, the upper set of lines correspond to the first-order solution, equation (53), and the lower set of lines to the second-order solution, equation (52). The symbols are numerical data obtained by the method of [5], where the approximation $h / R \ll 1$ is not made. In figure 2(b) the filled symbols correspond to $\rho g R^{2} / \gamma=2$ and the open symbols to $\rho g R^{2} / \gamma=-2$. The positive value is within the range of values taken by $\rho g R^{2} / \gamma$ in the experiments of Rodrigues et al. [4], as mentioned previously. 
It should be noted first of all that gravity has no effect on the analytical relation between $h / R, \theta_{1}$ and $\theta_{2}$, at least at the current order of approximation. This is a consequence of the fact that only the difference $p_{i 2}^{\prime}-p_{o 2}^{\prime}$ appears in equation (52), and according to equations (22) and (39), this difference does not depend on gravity, because the terms involving gravity cancel exactly.

On the other hand, the zeroth-order solution, which only takes into account the vertical curvature of the Plateau border, is trivial and a bad approximation: $\theta_{2}$ is not predicted to depend on $h / R$ or on $\theta_{1}$. The first-order approximation, where the horizontal curvature of the Plateau border is taken into account, produces a reasonable prediction for $\theta_{2}$, up to $h / R=$ 0.3, with the correct dependence on $\theta_{1}$. Finally, the second-order approximation, where apart from the physical effects mentioned above, the horizontal thickness of the Plateau border is taken into account, yields the best predictions. These are quite accurate up to $h / R \approx 0.45$. The analytical solutions nevertheless diverge considerably from the numerical results above these limits, as expected.

In figure 2(b), the numerical results show that, contrary to what the analytical model predicts, there is a dependence of $\theta_{2}$ on gravity, although this is relatively weak. For a given value of $h / R$, positive gravity (corresponding to a sessile bubble) decreases $\theta_{2}$, while negative gravity (corresponding to a pendant bubble) increases it.

Figure 2(c) compares the first-order and second-order analytical solutions and experimental data [4]. Note that this comparison is carried out over a much smaller range of $h / R$ than in figure 2(a,b). Experimentally, the bubbles are formed by blowing air through a hole in a porous glass substrate; the latter can be either 'dry', if only a few drops of surfactant solution are placed on it, or 'wet', if it is connected to the surfactant solution reservoir and therefore covered with a continuous liquid film [6]. Filled symbols are for bubbles on a 'dry' substrate, whereas open symbols are for bubbles on a 'wet' substrate. A contact angle of $\theta_{1}=0^{\circ}$ was assumed in either case, which seemed the most sensible choice, since the bubble was in contact with the fluid in both cases. The error bars were computed taking into account that the measurement error of $R$ is $0.05 \mathrm{~mm}$, and the error of $\theta_{2}$ is $1^{\circ}$ [4]. The measurement error of $h$ was estimated as $0.028 \mathrm{~mm}$ [6].

It can be seen that, while the agreement with the second-order analytical prediction is not very good (it is outside the error bars), the data approximately follow a straight line (which is consistent with the analytical results at any order of approximation for this range 
of $h / R$ ). However, the analytical results considerably overestimate $\theta_{2}$. The discrepancies might be attributed to a systematic measurement error, since the value of $h$ was estimated visually from the optical opacity of the Plateau border. It is plausible that the upper region of the Plateau border (which determines $h$ ) is very thin, and thus its upper limits are difficult to detect from mere visual inspection. This could account for an underestimation of $h$ (and thus also of $h / R$ ), although there may of course be other sources of error. We emphasize that these are the only experimental data that we know of which are relevant to the present problem.

\section{B. Extrapolated contact angle of the bubble}

The extrapolated contact angle of the bubble $\phi$ is the angle at which the bubble would intersect the substrate if the bubble's hemispherical shape extended for $z^{\prime}<1$. This angle is defined, by the use of standard trigonometry, as

$$
\cos \theta_{2}-\cos \phi=\frac{h}{R}
$$

Like $\theta_{2}, \phi$ does not depend on gravity at the current order of approximation. Owing to the way in which it is defined, $\phi$ is considerably closer to $90^{\circ}$ than $\theta_{2}$, with a narrower range of variation, and its relative error in the analytical approximation developed is thus expected to be larger.

In figure 3 the variation of $\phi$ with $h / R$ is presented for three values of $\theta_{1}$, for zero gravity (figure 3(a)), and for positive and negative gravity (figure 3(b)). Lines and symbols have the same meanings as in figure $2(\mathrm{a}, \mathrm{b})$. Taking into account equation $(56)$, it is clear that the zeroth-order value of $\phi$ is equal to the corresponding value of $\theta_{2}$, namely $\phi=90^{\circ}$, and therefore does not depend on $\theta_{1}$ or on $h / R$.

In figure 3(a), it can be seen that the first-order approximation for $\phi$ has reasonable accuracy up to $h / R=0.2$, and the second-order approximation is accurate up to about $h / R=0.4$. Both first-order and second-order approximations diverge strongly from the numerical results for larger values of $h / R$, but, again, this should be expected.

In figure 3(b), it can be seen that the dependence of $\phi$ on $h / R$ including gravity becomes inaccurate at approximately the same values of $h / R$ as in the zero-gravity case. However, discrepancies are slightly more marked for negative gravity (which is consistent with figure 
$2(\mathrm{~b}))$. For positive gravity, $\phi$ is somewhat smaller than in figure $3(\mathrm{a})$, staying closer to the prediction of the second-order approximation. In the case of negative gravity, on the contrary, $\phi$ is larger, and so departs more strongly from the second-order result. Qualitatively, this contrasting behaviour of a sessile and a pendant bubble (i.e., positive and negative gravity, respectively) appears intuitive, since a pendant bubble should portrude more from the substrate due to its own weight.

\section{Profiles of the Plateau border surfaces}

The solutions for $\Delta x^{\prime}$ and $z^{\prime}$ provided by equations (31)-(33) and (23)-(25) for the inner surface and by equations (47)-(49) and (40)-(42) for the outer surface are studied next for a bubble with $h / R \approx 0.4$. This large value of $h / R$ is chosen so that the effect of the second-order corrections is clearly visible, and on the other hand so that we are still within the limits of applicability of the theory (as suggested by figures 2 and 3).

Figure 4(a) shows the zeroth-, first- and second-order solutions for the shape of the Plateau border (with the bubble to the right). It is assumed that $\theta_{1}=10^{\circ}$ and $\theta_{2}=60^{\circ}$ (for the first- and second-order solutions), which corresponds to $h / R=0.408$ accurate to second-order in $h / R$ (and $h / R=0.436$ accurate to first-order). Also shown in figure 4(a) is a solution intermediate between the zeroth-order and the first-order solutions, which we have called $1 / 2$ th-order solution. This is equal to the zeroth-order solution, except that we have prescribed $\theta_{2}=60^{\circ}$ (as in higher-order solutions), which is inconsistent at zeroth-order (as equation (54) shows). The purpose of this is to understand more clearly the physical processes involved.

In figure $4(\mathrm{a})$ it can be seen that in the zeroth-order solution $\theta_{2}=90^{\circ}$ (as it must be) and the inner and outer surfaces of the Plateau border are symmetric arcs of circle. Relative to the zeroth-order solution, in the $1 / 2$ th-order solution the inner and outer surfaces of the Plateau border are translated to the left, due to the fact that $\theta_{2} \neq 90^{\circ}$. To consistenly be able to meet the substrate at the imposed value of $\theta_{1}$, the inner surface must increase its curvature considerably, whereas the outer surface must decrease it.

In the first-order solution, the additional effect of the horizontal curvature of the bubble is taken into account. This curvature has the same sign as the curvature of the inner surface, but the opposite sign to the curvature of the outer surface, increasing further the latter, but 
decreasing the former (for an approximately constant pressure inside the Plateau border). This effect is especially important when the surfaces are nearly vertical (i.e., near the top), while it is much weaker when they are nearly horizontal (i.e., near the bottom). This explains the slight translation of the surfaces to the left from the $1 / 2$ th- to the first-order solution. This effect is also stronger in the outer surface than in the inner surface, because the latter has larger curvature, and thus the relative effect of this correction is smaller. That explains the larger translation of the outer surface, and thus the slight widening of the Plateau border from the $1 / 2$ th- to the first-order solution. It is worth noting that although the changes on going from the zeroth-order to the $1 / 2$ th-order solution and from the $1 / 2$ th-order solution to the first-order solution are both of first-order (the former being associated with the vertical bubble curvature and the latter with the horizontal bubble curvature) in practice the former effect is much larger than the latter.

Changes on going from the first-order to the second-order solution are more subtle and difficult to interpret. These changes are due to the difference between distances of the inner and outer surfaces to the axis of symmetry (as was mentioned earlier), but this effect is not easy to understand in terms of curvature. It can nevertheless be noted that the secondorder solution is much closer to the first-order solution than the latter is to the zeroth-order solution. This suggests that the power series for $\Delta x^{\prime}$ and $z^{\prime}$ are asymptotic.

In figures 4(b,c), only results for the second-order solutions (the most accurate) are shown. Figure 4(b) displays the Plateau border surfaces for $\theta_{2}=60^{\circ}$ and $\theta_{1}=0^{\circ}, 10^{\circ}, 20^{\circ}$. This corresponds to $h / R=0.404, h / R=0.408$ and $h / R=0.417$, respectively, accurate to second-order. The Plateau border widens as $\theta_{1}$ decreases (i.e., as the fluid wets the solid increasingly better), being considerably larger for $\theta_{1}=0^{\circ}$ than for $\theta_{1}=20^{\circ}$. In figure $4(\mathrm{~b})$, agreement between the analytical and numerical solutions is excellent.

Finally, in figure 4(c), the dependence of the solutions on the gravity parameter is shown, again for a Plateau border with $\theta_{1}=10^{\circ}$ and $\theta_{2}=60^{\circ}$, corresponding to $h / R=0.408$. The effect of gravity is perhaps as expected, leading to a widening of the Plateau border for positive $\rho g R^{2} / \gamma$ (sessile bubble) and a narrowing for negative $\rho g R^{2} / \gamma$ (pendant bubble). This behaviour can be interpreted using hydrostatic equilibrium. For a positive $g$, the pressure is lower in the upper part of the Plateau border than in the lower part. This leads to an increase in the curvature of the inner and outer surfaces near the top, and a corresponding reduction near the bottom. It is straightforward to conclude that this 
corresponds to a widening. The converse happens for negative $g$.

The displacement of the outer surface of the Plateau border due to the variation of either $\theta_{1}$ or $g R^{2} / \gamma$ is much larger than the diplacement of the inner surface. This may be due, again, to the fact that the curvature of the inner surface is considerably larger, and thus less affected by higher order effects. Another possible cause is that the inner surface is closer to vertical, and thus a smaller horizontal translation is necessary for it to adjust to the variation of the parameters.

The agreement between analytical and numerical results, although qualitatively correct, is not so good for non-zero gravity. It might be that the values of $\rho g R^{2} / \gamma$ considered are too high, although this should not be the case, since they are of $O(1)$. We used these values so that the differences between the various curves in figure 4(c) were sufficiently clear (and also because the positive value is compatible with the experiments of [4], as remarked above). It should be recalled that gravity is treated here as a second-order effect, so when it becomes too large it would probably be better treated at lower order. But that approach, if feasible, would certainly increase the mathematical complexity further. A more fundamental mathematical reason for the worse performance of the analytical model including gravity may be the assumption (made in the description of the theoretical model, in section II) that $\theta$ is a monotonic function of $z$. When gravity effects are relatively large, the numerical simulations show that the curvature of the lower part of the Plateau border in a sessile bubble becomes weak and may even change its sign due to the hydrostatic increase of the pressure (not shown). In this case, $\theta$ stops being a monotonic function of $z$, and thus a one-to-one relation ceases to exist between these two variables (see figure 5).

\section{DISCUSSION}

It appears that, for an accurate prediction of the contact angle of relatively small bubbles (i.e., bubbles with sufficiently large $h / R$ ), it is crucial to consider at least a second-order approximation in our analytical, perturbation expansion, model. This physically corresponds to taking into account not only the horizontal curvature of the Plateau border (due to the sphericity of the bubble), but also the difference in distances to the axis of symmetry of the bubble from the inner and the outer surfaces of the Plateau border. Although gravity has a considerable impact on the shape of the Plateau border, which is captured by the 
present theory, its effect on the relation between $\theta_{2}, h / R$ and $\theta_{1}$ appears to be weak in most situations. These differing impacts of gravity on different aspects of Plateau border geometry seem to be corroborated by figures 2(b) and 4(c). Since bubbles with relatively high $h / R$ are generally small, and in that case $\rho g R^{2} / \gamma$ is generally low, the choice of treating gravity as a second-order effect may not be too inappropriate (it is in particular adequate for bubbles such as those studied experimentally by Rodrigues et al. [4]). However, in cases where gravity is more important, the calculations would need to be reformulated, for example with gravity considered at lower order in the perturbation expansion applied to the equations. Such calculations are, however, likely to be even more involved than those presented here. Numerical results also suggest that, when gravity effects are sufficiently strong, the curvature of the Plateau border surfaces may change sign locally (see figure 5). This would place a much more definite limit on the range of applicability of the present calculations, since they rely on the existence of a one-to-one relation between $\theta$ and $z$.

Within their limits of validity, the analytical calculations developed in the present paper have the advantage of providing closed-form expressions for the shape of the Plateau border, and an explicit relation between $\theta_{1}, \theta_{2}$ and $h / R$. This is a considerable improvement over the numerical model used to address a related problem [5], where numerical solutions must be integrated from the bottom of one of the Plateau border surfaces, and it is not possible to impose, for example, the coordinates of the upper vertex of the Plateau border. This limitation of the numerical model means that the contact angle of one of the Plateau border surfaces with the substrate must be adjusted by trial and error. Besides addressing the effect of gravity in a simple way, the analytical model proposed here therefore allows a much more exhaustive exploration of the parameter space than the numerical model.

\section{Acknowledgements}

We thank B. Saramago for illuminating discussions and an anonymous referee for insightful comments, which have considerably improved this paper. This work was supported by FCT under project AWARE/PTDC/CTE-ATM/65125/2006. 


\section{Dedication}

This paper is dedicated to the memory of M. A. Fortes, who initiated the work, but did not live to see its results.

[1] D. Weaire and S. Hutzler, Physics of Foams (Oxford University Press, Oxford, 1999).

[2] J. A. F. Plateau, Statique expérimentale et théorique des Liquides soumis aux seules Forces Moléculaires (Gauthier Villars, Paris, 1873).

[3] M. A. Fortes and P. I. C. Teixeira, "Excess energy of a plateau border at a wall," Phil. Mag. Lett. 85, 21 (2005).

[4] J. F. Rodrigues, B. Saramago and M. A. Fortes, "Apparent contact angle and triple-line tension of a soap bubble on a substrate," J. Colloid Interface Sci. 239, 577 (2001).

[5] P. I. C. Teixeira and M. A. Fortes, "Line tension and excess energy of a wall Plateau border," Phys. Rev. E 75, 011404 (2007).

[6] B. Saramago, private communication (2008). 


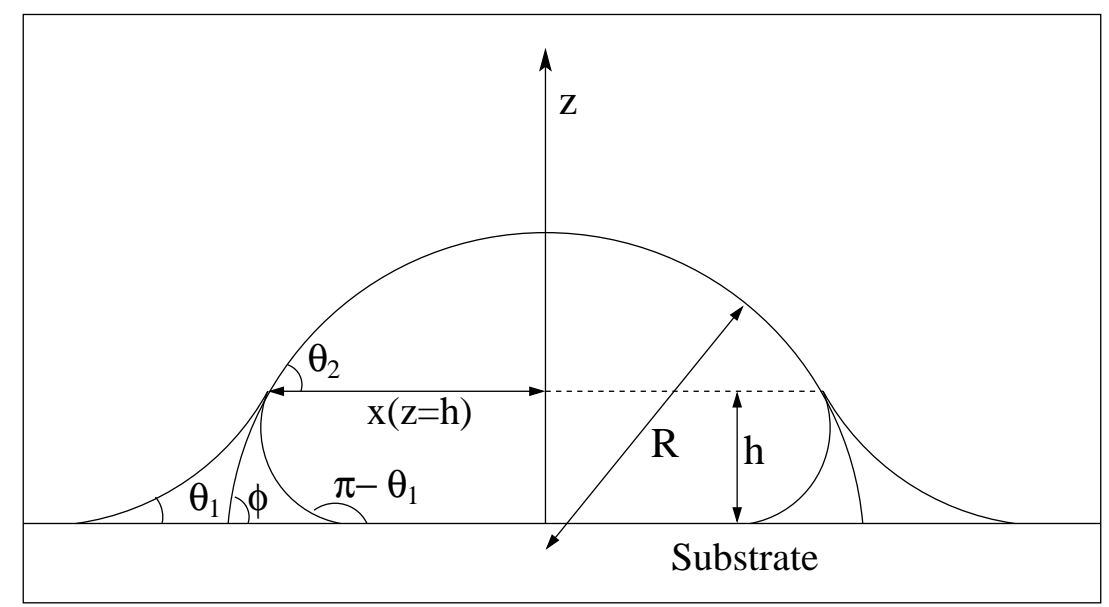

FIG. 1: Schematic diagram of the model problem. A bubble, and its associated Plateau border, are shown. $R$ is the radius of the bubble. $h$ is the height of the Plateau border. $\theta_{1}$ and $\pi-\theta_{1}$ are the contact angles of the Plateau border surfaces as they intersect the substrate. $\theta_{2}$ is the apparent contact angle of the bubble (angle of the film at the top of the Plateau border). $\phi$ is the extrapolated contact angle of the bubble in the absence of a Plateau border. $z$ is the height and $x$ is the radial distance of the film to its axis of symmetry (the $z$ axis). 

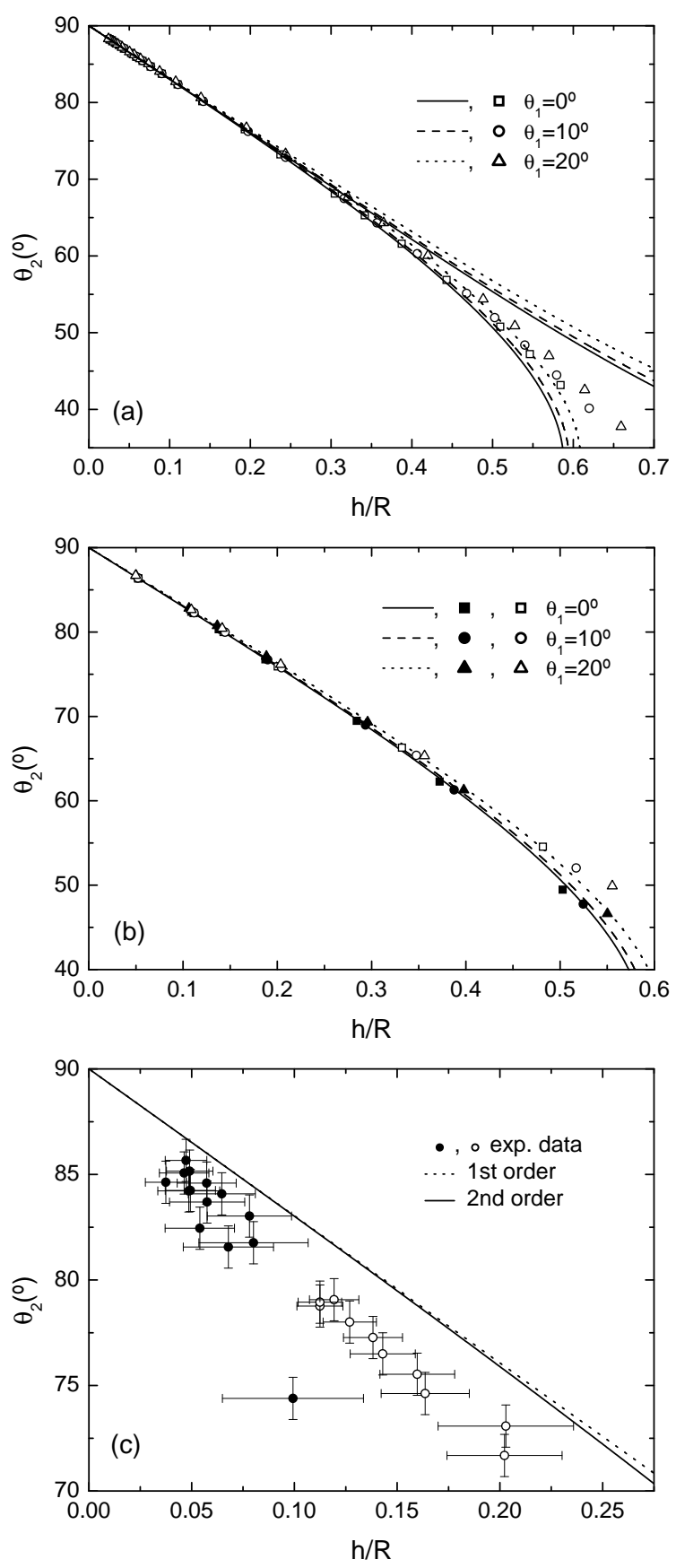

FIG. 2: Variation of the apparent contact angle of the bubble with the dimensionless height of the Plateau border. Lines: analytical model, symbols: numerical or experimental data. (a) Results without gravity. Upper set of lines: 1st-order approximation, lower set of lines: 2nd-order approximation. The 0th-order approximation coincides with the upper horizontal axis. Symbols: numerical model. (b) Results with gravity from 2nd-order approximation. Filled symbols: $\rho g R^{2} / \gamma=2$, open symbols: $\rho g R^{2} / \gamma=-2$, both from numerical model. (c) Comparison with experimental data (symbols) for $\theta_{1}=0^{\circ}$. The point at $\left(0.10,74^{\circ}\right)$ does not follow the general trend and may not be meaningful. 

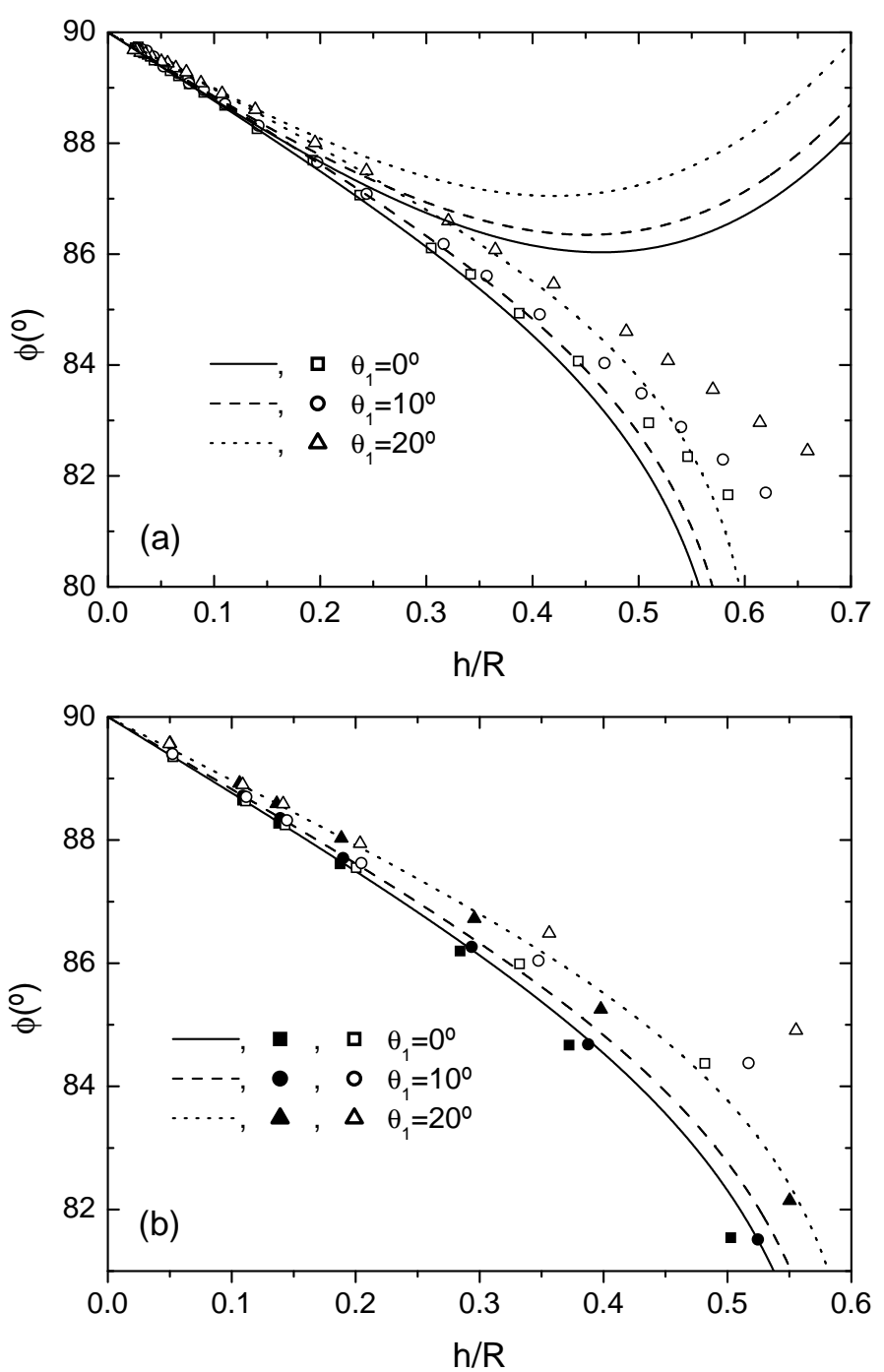

FIG. 3: Variation of the extrapolated contact angle of the bubble with the dimensionless height of the Plateau border. Lines: analytical model, symbols: numerical model. (a) Results without gravity. Upper set of lines: 1st-order approximation, lower set of lines: 2nd-order approximation. The 0th-order approximation coincides with the upper horizontal axis. (b) Results with gravity from 2nd-order approximation. Filled symbols: $\rho g R^{2} / \gamma=2$, open symbols: $\rho g R^{2} / \gamma=-2$. 

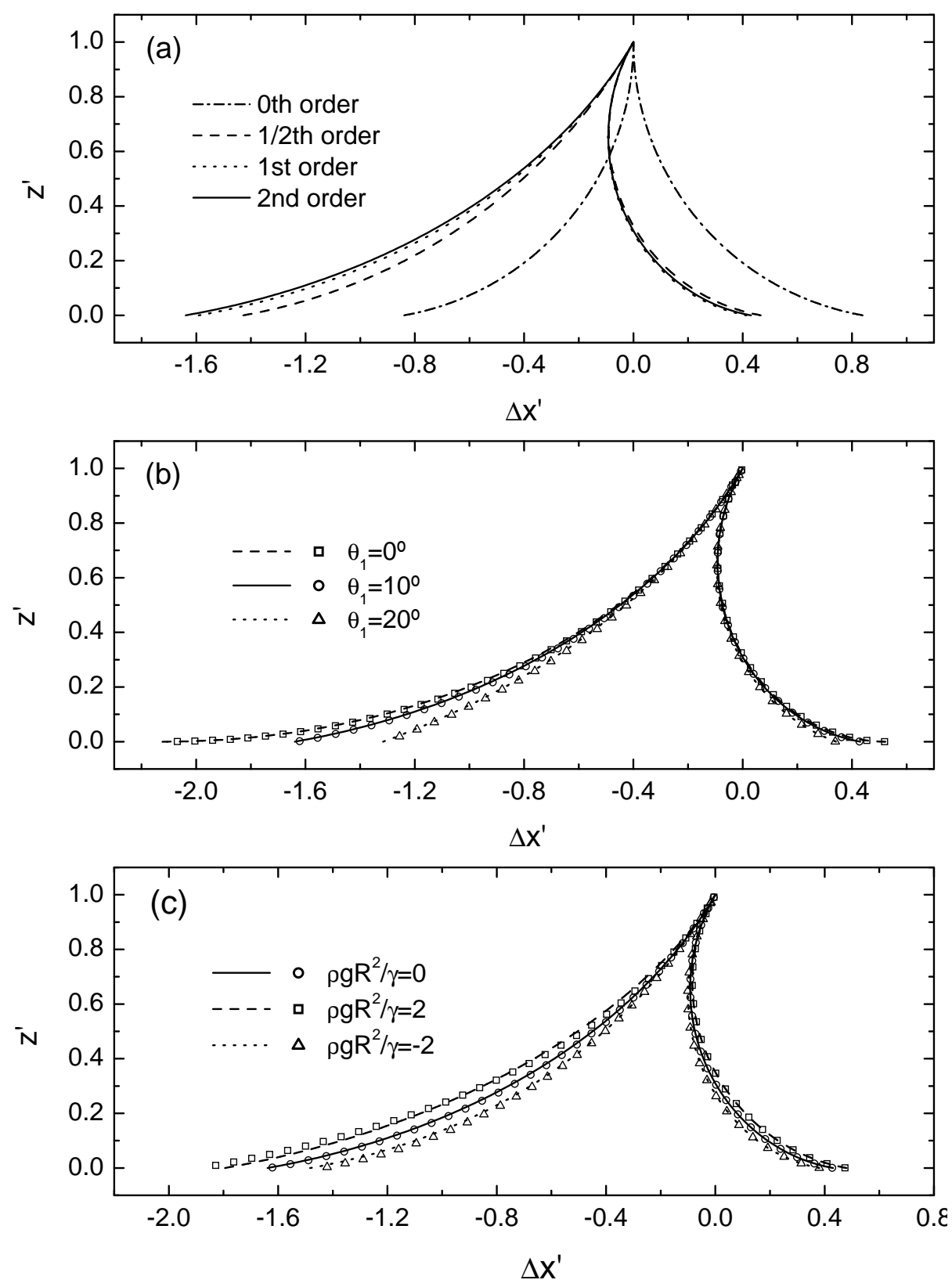

FIG. 4: Shapes of the outer (on the left) and inner (on the right) surfaces of the Plateau border for a bubble with $\theta_{2}=60^{\circ}$ (except for the 0th-order solution) $-h / R \approx 0.4$, near the limits of validity of the theory. Lines: analytical model, symbols: numerical model. (a) 0th-, 1/2th-, 1stand 2nd-order solutions for $\theta_{1}=10^{\circ}$, without gravity. (b) 2nd-order solution for $\theta_{1}=0^{\circ}, \theta_{1}=10^{\circ}$ and $\theta_{1}=20^{\circ}$, without gravity. (c) 2nd-order solution for $\theta_{1}=10^{\circ}$ and three values of $\rho g R^{2} / \gamma$. 


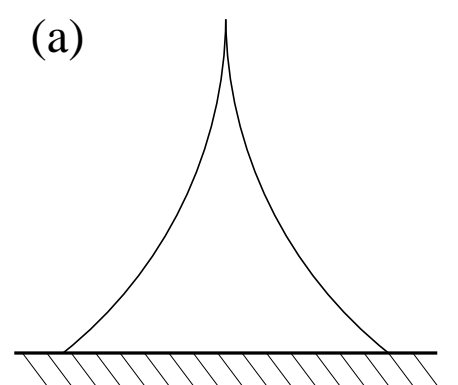

(b)

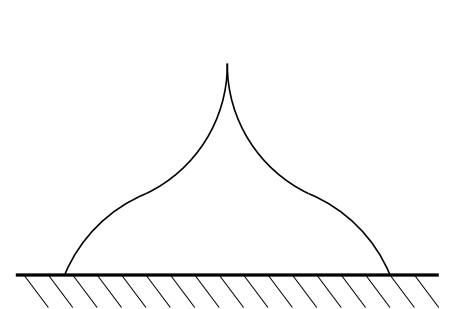

FIG. 5: Schematic diagram illustrating the approximate shape of the Plateau border of a sessile bubble with (a) weak gravity (b) strong gravity. The convex curvature at the bottom in (b) is due to the increase in pressure associated with hydrostatic equilibrium. 